\title{
Poor adherence to management guidelines in nontuberculous mycobacterial pulmonary diseases
}

\begin{abstract}
Nontuberculous mycobacterial pulmonary diseases (NTM-PD) are increasingly recognised as opportunistic infections of humans. Nodular/bronchiectatic disease and fibrocavitary disease are the most frequent manifestations [1]. Although guidelines have been published [1], there are few data on diagnostic and treatment practices outside of reference clinics. A recent survey in the USA suggested limited adherence to guidelines for diagnosis and treatment of NTM-PD [2].
\end{abstract}

We performed a survey on diagnostic and treatment practices for NTM-PD in five European countries (EU5) (France, Germany, Italy, Spain and the UK) and Japan, using previously published methods [2]. We randomly selected and contacted 3590 physicians (EU5: 2970; Japan: 620) from databases of each country [3, 4]; of these, 3154 (EU5: 2585; Japan: 569) participated. A total of 997 (EU5: 757; Japan: 240) physicians proved eligible for inclusion in round two, i.e. they managed at least one patient who 1) was diagnosed with NTM-PD, as defined by the participating physician; 2) was currently under the physician's care; 3) had active NTM-PD at some point during the past 12 months, regardless of whether treatment was provided; and 4) had complete information available. Of these eligible physicians, 619 (62\%; EU5: 446; Japan: 173) participated and entered data on 1429 NTM-PD cases, 1012 from the EU5 (France: 206; Germany: 211; Italy: 210; Spain: 230; UK: 155) and 417 from Japan. Most study physicians were pulmonologists (36\%; EU5: 29\%; Japan: 54\%), followed by internal medicine specialists (23\%; EU5: 21\%; Japan: 29\%) and general practitioners (23\%; EU5: 30\%; Japan: 5\%). They entered demographic, laboratory and treatment data from the patient records in an online questionnaire (33 questions with subquestions).

Mycobacterium avium complex (MAC) predominated as the causative agent of NTM-PD (EU5: 79.4\%; Japan: 85.1\%), followed by Mycobacterium abscessus (EU5: 20\%; Japan: 14.9\%). The patient groups were different in Japan and the EU5. Of all patients for whom information was entered in the EU5, 37\% were females, the mean age was $55.7 \pm 15.8$ years, $25 \%$ were never-smokers, $33 \%$ had chronic obstructive pulmonary disease (COPD) and $22 \%$ had bronchiectasis. In Japan, $65 \%$ of the patients were female, their mean age was $67.0 \pm 11.5$ years, $65 \%$ were never-smokers, $13 \%$ had COPD and $25 \%$ had bronchiectasis. Cough (86\%), sputum production (46\%), fatigue (52\%) and malaise (39\%) were the most frequently reported symptoms at diagnosis; fever was reported by $61 \%$ of the EU5 patients versus $26 \%$ of the Japanese patients.

Sputum sampling was the predominant microbiological diagnostic test for $63.5 \%$ of the EU5 patients and for $83.3 \%$ of the Japanese patients. Bronchial wash or lavage was performed in $52.6 \%$ of the EU5 patients versus $21.6 \%$ of the Japanese patients.

$71 \%$ of all patients reported from the EU5 had a (high-resolution) computed tomography (CT) scan to aid in the diagnosis of NTM-PD, ranging from $63.7 \%$ in the UK to $79.3 \%$ in Italy; in Japan, $80.5 \%$ of the patients had a CT scan. Chest radiographs were used for the diagnosis in $71.7 \%$ of all patients from the EU5 and $85.4 \%$ of all patients from Japan. CT revealed nodular/bronchiectatic disease in $39.2 \%$ of the EU5 patients, ranging from $33.2 \%$ in the UK to $45.5 \%$ in Spain, and in $59.8 \%$ of the Japanese patients.

Most patients in Japan were considered by their physicians to have mild disease (67\%; compared with $31 \%$ moderate, $2 \%$ severe), whereas most patients in the EU5 had moderate (59\%) or severe disease (19\%).

@ERSpublications

Poor adherence to diagnosis and treatment guidelines for nontuberculous mycobacterial lung disease in EU and Japan http://ow.ly/EVYl306vVcN

Cite this article as: van Ingen J, Wagner D, Gallagher J, et al. Poor adherence to management guidelines in nontuberculous mycobacterial pulmonary diseases. Eur Respir J 2017; 49: 1601855 [https://doi.org/ 10.1183/13993003.01855-2016]. 
In the EU5, $68 \%$ of the reported patients received antibiotic treatment for NTM-PD, whereas only $43 \%$ of patients reported from Japan received treatment. The percentage of patients receiving treatment differed by disease severity. $47 \%$ of patients with mild disease received treatment (EU5: 56\%; Japan: 40\%), versus 66\% (EU5: 70\%; Japan: 51\%) of patients with moderate disease and 77\% (EU5: 79\%; Japan: 29\%) of patients with severe disease. Symptomatic improvement was the most frequently cited goal of treatment (EU5: 46\%; Japan: 48\%), followed by sputum culture conversion (EU5: 31\%; Japan: 25\%).

Binary logistic regression analysis indicated that the country was the most influential factor for whether a patient was treated for NTM-PD. Patients in Spain and Italy are more than six times as likely to be treated than patients in France. Symptom severity and patient age were second and third most influential factors (table 1).

Oral antibiotics were the most frequently used treatment modality; for $27 \%$ of all patients, intravenous antibiotics were reported to be part of the treatment plan (EU5: 31\%; Japan: 11\%) and for 6\% of the patients inhaled antibiotics were reported to be part of treatment (EU5: 7\%, Japan: 1\%). Both intravenous and inhaled antibiotics were more frequently prescribed for M. abscessus than for MAC pulmonary disease (MAC-PD) (intravenous $42.5 \%$ versus $21.6 \%$; inhaled $11.7 \%$ versus $4.0 \%$ ).

For MAC-PD, patients mostly received macrolides (76\%), rifamycins (67\%), ethambutol (56\%) and fluoroquinolones (56\%; EU5: 45\%; Japan: 71\%). Only 16.9\% of all 746 treated MAC-PD patients received $>6$ months of a rifamycin-ethambutol-macrolide regimen: $41.9 \%$ of the treated patients in Japan but only $9.2 \%$ in the EU5. Within the EU5, this percentage differed by country: $17.8 \%$ in the UK, $8.4 \%$ in Spain, $8.0 \%$ in France, $7.6 \%$ in Italy and $4.3 \%$ in Germany. In the EU5, female patients were more likely than males to receive this regimen ( 25 out of 272 females versus 26 out of 740 males; $p=0.001$ ) and it was more often received by patients reported by pulmonologists (9\%) or infectious disease physicians (8\%) than by general practitioners $(4 \%)$ or general internal medicine physicians $(3 \%)$. In all participating countries, access to all these drugs is unrestricted, with physician prescription.

Oral antibiotic treatment for M. abscessus pulmonary disease involved use of macrolides (67\% of patients), fluoroquinolones $(54.3 \%)$, rifamycins $(32.5 \%)$, ethambutol (20\%), tetracyclines $(10 \%)$ and co-trimoxazole (5\%).

As only 9.2\% (EU5) and 41.9\% (Japan) of the treated MAC-PD patients received $>6$ months of the recommended rifamycin-ethambutol-macrolide regimen, published treatment guidelines seem to have had a limited impact on clinical practice. The EU5 data resemble those reported from the USA, where in a cohort of 915 patients, $80 \%$ had MAC-PD and only $13 \%$ of these MAC-PD patients received the guideline-compliant regimen [2]. These differences between the EU5, Japan and the USA could be related to the percentage of the reported patients seen by pulmonologists (54\% in Japan, $29 \%$ in the EU5), the

\begin{tabular}{lccc} 
TABLE 1 Variables influencing the treatment decision & & & \\
Variables & Odds ratio & Wald score & p-value \\
\hline Country & & 114.034 & 0.001 \\
$\quad$ Spain & 6.248 & 33.366 & 0.001 \\
Italy & 6.032 & 20.015 & 0.001 \\
UK & 4.005 & 2.960 & 0.085 \\
Germany & 2.665 & 2.910 & 0.088 \\
$\quad$ Japan & 1.441 & 2.346 & 0.126 \\
France (referent) & 1.000 & & \\
Physician-assessed symptom severity at presentation & & 8.229 & 0.016 \\
$\quad$ Severe & 1.932 & 6.750 & 0.001 \\
$\quad$ Moderate & 1.356 & 1.954 & 0.003 \\
$\quad$ Mild (referent) & 1.000 & & \\
Age & 1.018 & 6.275 & 0.012 \\
$\quad>60$ years & 1.641 & 5.205 & 0.023 \\
Sputum specimens for AFB analysis used in diagnosis & 1.420 & 6.819 & 0.009 \\
Fever a symptom at first presentation & 1.413 & 6.294 & 0.012 \\
Physician rating of patient's overall health & & 6.003 & 0.050 \\
$\quad$ Poor or very poor & 1.606 & 5.697 & 0.017 \\
$\quad$ Fair & 1.382 & 3.943 & 0.047 \\
$\quad$ Good or very good (referent) & 1.000 & &
\end{tabular}

AFB: acid-fast bacilli. ${ }^{\#}$ : exponentiation of the beta coefficient. 
level of acquaintance with the American Thoracic Society (ATS)/Infectious Diseases Society of America (IDSA) guidelines or availability of national guidelines promoting similar or different regimens, but this was not captured in the survey.

The predominance of nodular/bronchiectatic disease in Japan, as opposed to the EU5, probably drives the reported differences in disease severity and, as a result, the lower percentage of patients receiving treatment in Japan. Differences in likelihood of treatment within the EU5 may reflect the different rates of antibiotic consumption in European countries [5,6]. Less frequent use of CT scanning in the EU5 probably results from the predominance of fibrocavitary disease, but it may have hampered the recognition of nodular/ bronchiectatic NTM-PD [7].

$67 \%$ of all treated patients in the EU5 received a macrolide, compared with $98 \%$ in Japan and $43 \%$ in the USA [2]. Relatively few MAC-PD patients received a rifamycin and/or ethambutol. A regimen of ethambutol-clarithromycin in mild nodular/bronchiectatic disease proved non-inferior to rifampicin-ethambutol-clarithromycin in a recent study in Japan [8], so omitting the rifamycin might be an option for some patients. This may not hold true for ethambutol, as another recent study in Japan showed that not receiving ethambutol was an important risk factor for acquired macrolide resistance and failure [9]. Many patients used fluoroquinolones for both MAC-PD and M. abscessus pulmonary diseases, despite a lack of in vitro and in vivo efficacy [10-13]. The use of macrolide-quinolone regimens has been associated with the emergence of macrolide resistance [13].

In summary, proper diagnosis and treatment of NTM-PD require more attention by clinicians. Current treatment practices are likely to be ineffective and potentially hazardous, as they may induce further drug resistance. Significant educational efforts by professional societies are warranted to improve practices, starting with the implementation of the ATS/IDSA guidelines [1].

Jakko van Ingen $\oplus^{1,13}$, Dirk Wagner ${ }^{2,13}$, Jack Gallagher ${ }^{3}$, Kozo Morimoto ${ }^{4}$, Christoph Lange ${ }^{5,6,7,8}$, Charles S. Haworth ${ }^{9}$, R. Andres Floto ${ }^{9}$, Jennifer Adjemian ${ }^{10,11}$, D. Rebecca Prevots ${ }^{10}$ and David E. Griffith ${ }^{12}$ for the NTM-NET

${ }^{1}$ Dept of Medical Microbiology, Radboud University Medical Center, Nijmegen, The Netherlands. ${ }^{2}$ Division of Infectious Diseases, Dept of Medicine II, Medical Center - University of Freiburg, Faculty of Medicine, University of Freiburg, Freiburg, Germany. ${ }^{3}$ Clarity Pharma Research LLC, Spartanburg, SC, USA. ${ }^{4}$ Fukujuji Hospital, Japan Anti-Tuberculosis Association, Tokyo, Japan. ${ }^{5}$ Division of Clinical Infectious Diseases, Research Center Borstel, Borstel, Germany. ${ }^{6}$ German Center for Infection Research (DZIF), Clinical Tuberculosis Unit, Borstel, Germany. ${ }^{7}$ International Health/Infectious Diseases, University of Lübeck, Lübeck, Germany. ${ }^{8}$ Dept of Medicine, Karolinska Institute, Stockholm, Sweden. ${ }^{9}$ Cambridge Centre for Lung Infection, Papworth Hospital and Dept of Medicine, University of Cambridge, Cambridge, UK. ${ }^{10}$ National Institute of Allergy and Infectious Diseases, National Institutes of Health, Bethesda, MD, USA. ${ }^{11}$ United States Public Health Service, Commissioned Corps, Rockville, MD, USA. ${ }^{12}$ University of Texas Health Science Center, Tyler, TX, USA. ${ }^{13}$ Both authors contributed equally.

Correspondence: Jakko van Ingen, Dept of Medical Microbiology, Radboud University Medical Center, P.O. Box 9101, Nijmegen, 6500 HB, The Netherlands. E-mail: jakko.vaningen@radboudumc.nl

Received: June 132016 | Accepted after revision: Nov 082016

Support statement: This study was initiated and financed by Insmed Incorporated (Bridgewater, NJ, USA). The study was performed by Clarity Pharma Research LLC (Spartanburg, SC, USA) and the NTM-NET (Nontuberculous Mycobacteria Network European Trial Group). Insmed did not influence data acquisition or distribution. This work was supported in part by the Division of Intramural Research, National Institute of Allergy and Infectious Diseases (National Institutes of Health, Bethesda, MD, USA). Funding information for this article has been deposited with the Open Funder Registry.

Conflict of interest: Disclosures can be found alongside this article at erj.ersjournals.com

\section{References}

1 Griffith DE, Aksamit T, Brown-Elliot BA, et al. An official ATS/IDSA statement: diagnosis, treatment, and prevention of nontuberculous mycobacterial diseases. Am J Respir Crit Care Med 2007; 175: 367-416.

2 Adjemian J, Prevots DR, Gallagher J, et al. Lack of adherence to evidence-based treatment guidelines for nontuberculous mycobacterial lung disease. Ann Am Thorac Soc 2014; 11: 9-16.

3 Organisation for Economic Co-operation and Development (OECD). OECD Health Statistics 2014 - Frequently Requested Data. www.oecd.org/els/health-systems/oecd-health-statistics-2014-frequently-requested-data.htm Date last accessed: September 20, 2016. Date last updated: November 2014.

4 European Pharmaceutical Marketing Research Association (EphMRA). Doctor Statistics in Canada, France, Germany, Italy, Japan, Spain, UK, USA. London, EphMRA, 2008. Available from: www.ephmra.org/user_uploads/ ephmra\%20dr\%20stats\%20report\%20final\%204\%20april\%2008(1).pdf

5 Adriaenssens N, Coenen S, Versporten A, et al. European Surveillance of Antimicrobial Consumption (ESAC): outpatient antibiotic use in Europe (1997-2009). J Antimicrob Chemother 2011; 66: Suppl. 6, vi3-vi12.

6 Ferech M, Coenen S, Malhotra-Kumar S, et al. European Surveillance of Antimicrobial Consumption (ESAC): outpatient antibiotic use in Europe. J Antimicrob Chemother 2006; 58: 401-407.

7 van Ingen J, Bendien SA, de Lange WC, et al. Clinical relevance of non-tuberculous mycobacteria isolated in the Nijmegen-Arnhem region, The Netherlands. Thorax 2009; 64: 502-506. 
8 Miwa S, Shirai M, Toyoshima M, et al. Efficacy of clarithromycin and ethambutol for Mycobacterium avium complex pulmonary disease. A preliminary study. Ann Am Thorac Soc 2014; 11: 23-29.

9 Morimoto K, Namkoong H, Hasegawa N, et al. Macrolide-resistant Mycobacterium avium complex lung disease: analysis of 102 consecutive cases. Ann Am Thorac Soc 2016; 13: 1904-1911.

10 Jeon K, Kwon OJ, Lee NY, et al. Antibiotic treatment of Mycobacterium abscessus lung disease: a retrospective analysis of 65 patients. Am J Respir Crit Care Med 2009; 180: 896-902.

11 Koh WJ, Hong G, Kim SY, et al. Treatment of refractory Mycobacterium avium complex lung disease with a moxifloxacin-containing regimen. Antimicrob Agents Chemother 2013; 57: 2281-2285.

12 van Ingen $\mathrm{J}$, Egelund $\mathrm{EF}$, Levin $\mathrm{A}$, et al. The pharmacokinetics and pharmacodynamics of pulmonary Mycobacterium avium complex disease treatment. Am J Respir Crit Care Med 2012; 186: 559-565.

13 Griffith DE, Brown-Elliott BA, Langsjoen B, et al. Clinical and molecular analysis of macrolide resistance in Mycobacterium avium complex lung disease. Am J Respir Crit Care Med 2006; 174: 928-934.

The content of this work is not subject to copyright. Design and branding are copyright @ERS 2017 